\title{
Autoridad epistémica y atenuación en Tena Kichwa: Análisis de enclítico =cha basado en el corpus
}

EPISTEMIC AUTHORITY AND ATTENUATION IN TENA KICHWA:CORPUS-BASED ANALYSIS OF THE ENCLITIC $=C H A$

\section{Karolina GRZECH}

SOAS, Universidad de Londres

Resumen:

Este artículo se enfoca en la relación entre atenuación y falta de autoridad epistémica. Los datos analizados en el artículo provienen de tena kichwa, una lengua quechua en peligro de extinción, hablada en la Amazonia Ecuatoriana. La lengua posee un paradigma de marcadores que son cognados de los marcadores evidenciales en otras lenguas quechuas, pero que en tena kichwa se relacionan con la autoridad epistémica: 'el derecho del hablante a saber o sostener algo' (Stivers et al. 2011).

El artículo describe la semántica de los dos marcadores de autoridad epistémica en tena kichwa: =mi y =cha. Se concentra sobre todo en los efectos de atenuación que se pueden lograr a través del uso de =cha. Propone que el marcador, que codifica 'la falta de autoridad epistémica del hablante', puede tener un sutil efecto atenuativo, ya que 'distancia al hablante de la elocución' y 'reduce la fuerza comunicativa de la oración (Briz y Albelda 2013). Destaca que este efecto atenuador no consiste en reducir el compromiso del hablante con la veracidad de la aseveración. Por lo mismo, el análisis atenuativo de =cha en tena kichwa abre preguntas teóricas interesantes en cuanto a lo que se puede considerar una estrategia de atenuación en el discurso.

Palabras clave: quechua, tena kichwa, atenuación, evidencialidad, primacía epistémica, marcadores del discurso.
Abstract:

This article focuses on the relationship between attenuation and lack of epistemic authority. It draws on the data from Tena Kichwa, an endangered Quechuan language spoken in the Ecuadorian Amazon. Tena Kichwa has a paradigm of markers which are cognates of evidential markers in other Quechuan varieties, but which in this language are related to epistemic authority: 'the speaker's right to know or claim' (Stivers et al. 2011).

The article describes the semantics of the two 'epistemic authority' markers, =mi and =cha, and focuses on the discursive effect of attenuation which can be achieved through the use of $=c h a$. It proposes that through encoding 'lack of epistemic authority' =cha achieves the a subtle attenuative effects of 'distancing speakers from their statements' and 'reducing the communicative force of an utterance' (Briz y Albelda 2013). Interestingly, this effect does not consist in reducing the speaker's commitment to the veracity of the proposition. Thus, the attenuative analysis of the Tena Kichwa =cha opens some interesting questions about the conceptual boundaries of what can be considered as an strategy for linguistic attenuation.

Key words: Quechua, Tena Kichwa, attenuation, evidentiality, epistemic primacy, discourse markers 


\section{normas}

\section{INTRODUCCIÓN}

El tema principal de este artículo es el rol del enclítico =cha en los procesos de atenuación en tena kichwa, una lengua quechua hablada en la Amazonía Ecuatoriana. El contenido semántico del enclítico =cha tiene que ver en tena kichwa con la falta de la autoridad epistémica del hablante (véase Heritage y Raymond 2005; Stivers et al. 2011), y - en otros dialectos quechuas - con evidencialidad inferencial y conjetural (véase entre otros: Weber 1986; Floyd 1997; Faller 2002).

A lo largo de este trabajo, 'atenuación' se entiende como una 'actividad argumentativa (retórica) estratégica de minimización de la fuerza ilocutiva y del papel de los participantes en la enunciación' (Briz y Albelda 2013: 292). ${ }^{1}$ En las lenguas europeas, propiedades atenuativas se atribuyen frecuentemente a expresiones asociadas con la modalidad epistémica, ya que estas reducen el grado de certeza del hablante con respecto a la aseveración (véase entre otros Palmer 2001).

El objetivo del presente artículo es explorar el rol de marcadores epistémicos que expresan otros valores que la modalidad epistémica en el proceso de atenuación. Este fin se logra a través del estudio de caso del marcador epistémico =cha en tena kichwa. Dicho marcador indica que el hablante considera que una aseveración se encuentra fuera de su 'territorio de información' (véase Kamio 1997), y que antes de que pueda considerarse como verdadera por parte de todos los participantes del discurso, se requiere una elaboración adicional por parte del interlocutor. El presente artículo se enfoca en explicar cómo la semántica epistémica de =cha se usa en el discurso para lograr el efecto atenuador. Antes de presentar la orden de exposición que voy a seguir en el presente trabajo, cabe introducir brevemente la lengua tena kichwa y explicar el proceso de recaudación de datos.

Tena kichwa es una lengua quechua hablada en la Amazonia Ecuatoriana, que pertenece al grupo Qll de los dialectos quechuas (Torero 1964). Las fuentes más recientes indican entre 20 y 40 mil hablantes (Moseley 2010; Lewis 2016). ${ }^{2}$ Como las demás lenguas quechuas, tena kichwa es una lengua aglutinante, que usa casi exclusivamente sufijos. ${ }^{3}$ En las lenguas quechuas predomina por lo general la orden SOV (Sujeto-Objeto-Verbo), aunque está regla se aplica más estrictamente a las oraciones subordinadas que a las cláusulas principales (veáse Muysken 1995; Adelaar y Muysken 2004). En tena kichwa, la orden SOV es la única correcta en las oraciones subordinadas, pero en las clausulas principales SOV se usa con una frecuencia muy similar al SVO (véase Grzech 2016: cap. 4).

\footnotetext{
'Es importante destacar que atenuación y cortesía no son el mismo fenómeno; menos atenuación no implica necesariamente un menor grado de cortesía (Briz y Albelda 2013: 296).

${ }^{2}$ Tena kichwa está en contacto intenso con el castellano, la lengua oficial de Ecuador. Tanto kichwa, como shuar (jíbara) están considerados como 'idiomas oficiales de relación intercultural' (ANCE 2008), y gozan de un estatus semioficial en el país. No obstante, el uso del kichwa está disminuyendo, sobre todo en las comunidades cercanas a los mayores centros poblacionales. Las inversiones en infraestructura y la presencia de compañías petrolíferas afectan la movilidad y posibilidades laborales de los hablantes del tena kichwa, contribuyendo a la interrupción de la transmisión intergeneracional.

${ }^{3}$ El único prefijo atestiguado en la documentación es la-, usado para distinguir los miembros de la familia política de la familia biológica, por ejemplo mama 'mamá, madre' vs. lamama 'madrastra'.
} 
Los datos que hicieron posible este trabajo han sido recaudados dentro de un proyecto de documentación de tena kichwa. Dentro del proyecto, realicé casi un año de trabajo de campo en la provincia de Napo, en Ecuador, entre 2013 y 2014. ${ }^{4}$ El proyecto de documentación que se realizó durante este periodo ha sido colaborativo. Los miembros de la comunidad kichwa de Nuevo Paraíso han seleccionado los temas a documentar, han actuado como camarógrafos y entrevistadores. Además, han transcrito en kichwa y traducido al castellano cerca de 23 horas de datos de audio y vídeo recopilados dentro del marco del proyecto. La documentación del idioma y de temas de importancia cultural ha sido acompañada de sesiones de grabaciones que sirvieron para explicar algunos aspectos de la gramática de tena kichwa, y de sesiones experimentales. El trabajo presentado en las siguientes páginas se basa en un corpus de 13 horas, que incluye 11 horas del discurso natural (monólogos, entrevistas, discurso político, eventos), y 2 horas del discurso semi-natural, obtenido dentro del marco de las grabaciones de experimentos lingüísticos.

El presente artículo está organizado en seis secciones principales. La presente sección, introduzco el tema del artículo, y la lengua analizada a continuación. En la siguiente sección, defino las principales nociones relevantes para el análisis del enclítico =cha. Por consiguiente, describo la semántica de los sistemas evidenciales quechuas y explico cómo se sitúa el sistema 'evidencial' de tena kichwa con respecto a las otras lenguas de la misma familia. A continuación, me enfoco en =cha, demostrando su uso discursivo y su rol en el proceso de atenuación lingüística en tena kichwa. Finalmente, propongo algunas conclusiones con respecto a la relación entre las expresiones de autoridad epistémica y el proceso discursivo de atenuación.

\section{DEFINICIONES}

Para poder desarrollar la análisis propuesta en la sección anterior, es necesario definir algunas nociones teóricas que se usará na a lo largo de este trabajo. La noción de 'atenuación' ha sido definida en la introducción. En esta sección me concentraré en definir los dos términos de principal importancia para describir la semántica del =cha, incluyendo 'evidencialidad', 'epistemicidad' y 'autoridad epistémica'.

La evidencialidad se entiende en las siguientes páginas como codificación lingüística del modo de acceso a la información (Izquierdo Alegría y Cornillie 2017). Por lo tanto, evidencialidad se entiende como una categoría semántica y funcional distinta a la modalidad epistémica, la cual tiene que ver con la certeza sobre la veracidad de la información (véase Palmer 2001). Sin embargo, la separación conceptual entre la evidencialidad y la modalidad epistémica no significa que no existan lenguas en las cuales las dos categorías estén indicadas por el mismo paradigma morfosintáctico. De hecho, en

\footnotetext{
${ }^{4}$ Agradezco a todos los habitantes de Nuevo Paraíso. Sin su colaboración y acogida la investigación descrita en este artículo nunca hubiera podido realizarse. Sobre todo agradezco a mis colaboradores y amigos, quienes crearon junto a mí el proyecto de documentación: Nilo Andy, Wilma Aguinda y Jacobo Chimbo. También doy las gracias a los demás colaboradores del proyecto. Agradezco a Lidia Chimbo y su familia por acogerme, y a Yolanda Chimbo por su amistad y por las risas que hemos compartido.

Este proyecto ha sido financiado por Endangered Languages Documentation Programme (número de beca IGS00166) y también ha recibido apoyo de Frederick Soddy Trust. La documentación está depositada en Endangered Languages Archive (https://elar.soas.ac.uk/Collection/MPI849403). Se está preparando el depósito para el Archivo de Lenguas y Culturas del Ecuador de FLACSO Andes (http://www.flacsoandes.edu.ec/lenguas-culturas/).
} 


\section{normas}

Autoridad epistémica y atenuación en Tena Kichwa | Karolina Grzech

varios idiomas utilizan paradigmas polisémicos que abarcan las dos categorías (véase entre otros Aikhenvald 2004; Boye 2012).

Boye (2012) entiende la evidencialidad y la modalidad epistémica como dos sub-categorías de la noción de 'epistemicidad'. Según Boye (2012), tanto evidencialidad como modalidad epistémica contribuyen al 'respaldo justificativo' de una proposición. La evidencialidad contribuye 'justificación epistémica', directa o indirecta (Boye 2012: sec. 5.1.3). La modalidad epistémica, por otra parte, contribuye 'respaldo epistémico' (Boye 2012: 36), sea 'pleno' (certidumbre), 'parcial' (probabilidad) o 'neutro' (cuando una proposición no contiene calificación epistémica). Sin embargo, en este artículo adopto una definición más amplia de 'epistemicidad', según la cual dicha categoría semántica/funcional no se limita sólo a la evidencialidad y la modalidad epistémica, sino también puede contener otros tipos de significados asociados con las diferentes dimensiones de lo que significa 'saber' (véase Bergqvist 2017).

Uno de tales significados es la 'autoridad epistémica', relacionada con 'el derecho relativo a saber o sostener algo' (Stivers et al. 2011: 11). Cabe destacar que la autoridad epistémica es más subjetiva que la evidencialidad. En el caso de la última, el modo de acceso a la información, sea visual, inferencial o reportativo, relaciona al hablante con el mundo extradiscursivo. En cambio la autoridad epistémica, si bien puede estar relacionada con como el hablante ha adquirido la información, es más subjetiva. El hablante puede sentirse en derecho de hacer ciertas aseveraciones en función de quien es su interlocutor, o de su evaluación de lo que el interlocutor sabe o cree. Por lo tanto, la autoridad epistémica puede considerarse incluso intersubjetiva (véase por ejemplo Traugott 2010), ya que al expresarla, el hablante tiene que considerar lo que sabe o no sabe su interlocutor.

En la literatura reciente, se empiezan a usar nociones nuevas, relevantes a la autoridad epistémica. Uno de ellos es 'el desbalance epistémico' (Mushin 2017), que se refiere al hecho de que existe un desequilibrio de conocimiento entre el hablante y el oyente. Como voy a demonstrar a continuación, =cha en tena kichwa marca un tal desbalance, indicando que el interlocutor puede saber más que el hablante. Otra noción es la del 'compromiso' (engagement, véase Evans et al. En Prensa), que tiene que ver con la 'relativa accesibilidad de un referente para el hablante y el oyente'. Cuanto más 'comprometido' el hablante, más grande es su autoridad epistémica.

Otro concepto asociado tanto con el 'compromiso' como con la autoridad epistémica, y que ayuda a entender las dos nociones, es el 'territorio de información' (Kamio 1997): ‘la información que el hablante considera como próxima a sí mismo, en el sentido de que no hubiera sido natural para otro hablante usarla, remplazando yo con tu' (Kamio 1997: 2). Un ejemplo típico de información que se posiciona dentro del territorio de información de cada persona es la información sobre sus sentimientos, su familia, lo que se llama de manera coloquial 'información personal', o información relacionada con la pericia profesional de uno.

El objetivo de este artículo es explicar cómo la autoridad epistémica se relaciona con la atenuación en tena kichwa. Sin embargo, antes de empezar el análisis del uso del enclítico =cha como herramienta de atenuación, cabe describir la posición que ocupa dicho 


\section{normas}

Autoridad epistémica y atenuación en Tena Kichwa | Karolina Grzech

marcador en el sistema morfosintáctico del tena kichwa y su contribución semántica a la oración.

\section{LOS ENCLÍTICOS - MARCADORES DEL DISCURSO EN TENA KICHWA}

Como se ha mencionado anteriormente, tena kichwa y las demás lenguas quechuas son aglutinativas, con la orden predominante de SOV, y utilizan casi exclusivamente sufijos. Las dos principales categorías gramaticales del Quechua son los verbos y los substantivos; los sufijos se dividen entre verbales y nominales, dependiendo de la principal categoría gramatical con la que ocurren. Sin embargo, las lenguas quechua, incluyendo el tena kichwa, también tienen una clase de enclíticos que pueden ocurrir con palabras de todas las categorías gramaticales que pueden funcionar como núcleos sintácticos: verbos, substantivos, adverbios, adjetivos predicativos etc.

Los datos recaudados dentro del proyecto de documentación del tena kichwa muestran que en el idioma existen quince enclíticos. Entre ellos, nueve pueden analizarse como 'enclíticos discursivos' (véase Spencer y Luis 2012), ya que su distribución está condicionada no por reglas sintácticas, sino por factores relacionados con el discurso. Por lo tanto, el hecho de no usar uno de los enclíticos discursivos no hace que la oración sea gramaticalmente incorrecta. Más bien, el hecho de usar los enclíticos discursivos hace que la oración puede o no ser apropiada según el contexto comunicativo en el que ocurra. Por lo tanto, este grupo de enclíticos pueden considerarse como marcadores de discurso (véase entre otros Schiffrin 1987; Maschler y Schiffrin 2015), ya que contribuyen a la organización del discurso y a las relaciones entre los participantes, y no al contenido proposicional de lo enunciado.

La morfosintaxis y la semántica básica de todos los enclíticos en tena kichwa se describen con más detalle en Grzech (2016: cap. 3). Ahí también se aclara la contribución que hace cada uno de los enclíticos al nivel del discurso. En este artículo abordaré solo dos enclíticos discursivos - =mi y =cha $-\mathrm{y}$ me enfocaré sobre todo en el segundo de ellos.

En las descripciones de otros dialectos quechuas, =mi ha sido analizado como un marcador validacional (Adelaar 1977; Cole 1982), un evidencial directo (Weber 1986; Floyd 1997; Faller 2002), el marcador del 'mejor posible fundamento' (Best Possible Ground) para una aseveración (véase Sección 4) y como un marcador de foco (Muysken 1995; Cusihuamán 1976/2001; Sánchez 2010). Los cognados de =cha han sido analizados como: un marcador validacional (Adelaar 1977; Cole 1982), evidencial indirecto conjetural e inferencial (Weber 1986; Floyd 1997), evidencial indirecto conjetural e inferencial y marcador modal epistémico (Faller 2002).

A continuación demuestro que ninguno de los análisis mencionados anteriormente refleja de manera adecuada la semántica de $=m i \mathrm{y}=$ cha en tena kichwa. También expongo un análisis alternativo, según el cual la función principal de dichos marcadores en tena kichwa es indicar la (falta de) autoridad epistémica del hablante.

\section{EL SISTEMA EVIDENCIAL EN LAS LENGUAS QUECHUAS Y EN TENA KICHWA}

En esta sección describo el paradigma evidencial atestiguado en la mayoría de los dialectos documentados del quechua (4.1). Por consiguiente, comparo y contrasto este paradigma 
con el sistema del tena kichwa, con el fin de explicar las funciones discursivas de los enclíticos 'evidenciales' presentes en esta variante lingüística (4.2).

\subsection{Paradigma evidencial quechua}

Antes de que se estableciera el análisis evidencial, los marcadores en cuestión han sido analizados como 'comentario de cláusula' (Parker 1969) o marcadores validacionales que indican 'el juicio epistémico del hablante' (Adelaar 1977; Cole 1982; Lefebvre y Muysken 1988). El análisis evidencial ha sido propuesto por Weber (1986) en su descripción de los enclíticos en huánuco quechua (Ql) y desde entonces ha sido adaptado para la descripción de los enclíticos en muchas otras lenguas quechuas (véase entre otros Floyd 1997; Faller 2002; Hintz 2012; Howard 2012).

Sin embargo, los análisis anteriores no han sido completamente descartados. En los diferentes dialectos, los 'evidenciales' quechuas indican no sólo el modo de acceso a la información, sino también: el juicio epistémico del hablante/origo (Weber 1986; Nuckolls 1993; Floyd 1997; Adelaar 1997), la fuerza ilocutiva (Faller 2002; Nuckolls 2012), la subjetividad del hablante (Howard 2012; Nuckolls 2012), o la diferencia entre el conocimiento individual y el conocimiento compartido (Howard 2012; Hintz y Hintz 2017). En algunos dialectos, ciertos enclíticos se han analizado como 'puramente' evidenciales, y otros se han considerado más validacionales (Adelaar 1977; Faller 2002).

La mayoría de las lenguas quechuas descritas hasta el momento hacen una división evidencial entre tres modos de acceso a la información: directa, inferencial/ conjetural y reportativa.

a. Evidencial directo/'mejor fundamento posible’: =mi

Parashanmi.

para -sha $-\mathrm{n}=\mathbf{m i}$

llover-PROG-3=mi

'Está lloviendo.'

Contexto: El hablante ve que está lloviendo.

b. Evidencial inferencial/conjetural: =chá

Parashanchá.

para -sha -n=chá

llover-PROG-3=chá

'Está lloviendo.'

Contexto: El hablante conjetura que está lloviendo.

c. Evidencial reportativo: $=s i$

Parashansi.

para -sha $-\mathrm{n}=\mathbf{s i}$ 


\section{normas}

Autoridad epistémica y atenuación en Tena Kichwa | Karolina Grzech

llover-PROG-3=si

'Está lloviendo.'

Contexto: Al hablante le han dicho que está lloviendo.

Adaptado de Faller (2002: 122)

Para explicar el sistema evidencial quechua expuesto anteriormente, cabe elucidar en más detalle la semántica evidencial que respalda las etiquetas asignadas a los marcadores en cuestión. Ya que el marcador reportativo no ocurre en tena kichwa, en lo que sigue me concentraré en el concepto de evidencialidad directa y 'el mejor fundamento posible' y en la evidencialidad inferencial/conjetural.

Para poder explicar el concepto de 'mejor fundamento posible', introducido por Faller (2002) es necesario primero describir en detalle el concepto de evidencialidad directa. Este tipo de evidencialidad requiere que el hablante tenga 'acceso directo' a la información descrita en la oración (véase entre otros Willett 1988; Aikhenvald 2004). Sin embargo, lo que constituye el 'acceso directo' puede variar según el tipo de evento en cuestión. Tal acceso puede obtenerse a través de los sentidos, mayormente - la vista ('Acaba de salir el sol'). No obstante, también existen eventos que pueden apreciarse de manera directa a través de los otros sentidos, tales como tacto ('La piedra está caliente') u oído ('Los niños están cantando desentonadamente'), o bien, en el caso de sentimientos y sensaciones internas como tristeza o felicidad, el acceso directo no requieres acceso sensorial. Por lo tanto, el 'acceso directo' parece ser una 'característica graduable de eventos' (Faller 2002: 131). Faller (2002: 131-34) distingue entre acciones/hechos observables (comer, ser alto), y no-observables (estados internos, pensamientos). Determinar lo que constituye el acceso directo a hechos observables es relativamente simple, pero en el caso de eventos noobservables es necesario considerar casa caso individualmente. Además, como menciona Faller (2002:134), existen aseveraciones para las cuales es imposible determinar de dónde proviene la información. Tal caso son las convicciones religiosas ('Dios existe').

Determinar si el acceso a la información es directo es solo uno de los elemento necesarios para establecer si el hablante posee 'el mejor fundamento posible' para una aseveración. El otro componente de la semántica del 'mejor fundamento' es el tipo de conocimiento en el que se basa la aseveración. Faller (2002: 133-35) distingue entre el 'conocimiento personal' y 'conocimiento enciclopédico'. Conocimiento personal abarca la experiencia de vida del hablante, y todos los hecho que este ha experimentado personalmente. La segunda categoría se refiere a conocimiento al que 'se toma por sentado dentro de una cultura, y conocimiento normalmente enseñado en escuelas e incluido en enciclopedias' (Faller 2002: 133). Un ejemplo podría ser el conocimiento sobre los tamaños y orden relativos al sol de los diferentes planetas de nuestro sistema solar. No hemos obtenido esta información a través de nuestros sentidos, pero lo integramos plenamente dentro de un conjunto de saberes que tenemos sobre el mundo, junto a la información sobre aquellos hechos que podemos observar directamente.

Teniendo en cuenta estos diferentes tipos de conocimiento, Faller (2002) indica que en cuzco quechua, =mi tiene un significado más amplio que 'fuente directa de información' y que debe más bien analizarse como un marcador de 'mejor fundamento posible' para una aseveración. El 'mejor fundamento posible' equivale a una evidencia directa en el caso de 
los conocimientos que provienen de la vida personal del hablante, pero puede incluir una evidencia reportativa en el caso de conocimiento enciclopédico (Faller 2002: 135). Según Faller, para usar el marcador =mi de forma exitosa, el hablante necesita (1) tener la mejor fuente de información posible para el determinado tipo de evento, (2) estar seguro/a de tener la mejor fuente de información, y (3) haber integrado la información marcada con =mi en su el sistema de conocimientos sobre el mundo (Faller 2002: 140-1). En la Sección 4.2 muestro que esta interpretación de $=m i$ no permite explicar todos sus usos atestiguados en el tena kichwa.

El segundo enclítico que aparece es =cha: evidencial inferencial y conjetural, en el que se enfoca este artículo. Según Faller (2002), =cha en cuzco quechua es a la vez un evidencial y un modal epistémico. El significado evidencial de =cha es indicar que el hablante 'basa su oración en un proceso mental', sea conjetura, inferencia, adivinanza o cualquier otro proceso que supone el uso de la razón (Faller 2002: 176). Sin embargo, Faller también indica que en el caso de que el hablante base su oración en la evidencia directa, pero parcial, no se usa =cha, sino una partícula -chus hina, que ocurre en distribución complementaria con los otros marcadores evidenciales en cuzco quechua. El ejemplo (1) muestra tanto el uso de =cha como el de =chus hina:

(1)

Contexto: Marya se ve muy pálida.
a. ?Unqusqachá
kashanman.
unqu-sqa-chá ka-sha-n-man
enfermo-PRT-CONJ ser/estar-PROG-3-COND
'Puede que esté enferma.'
b. Unqushkachus hina kahanman.
unqu-sqa-chus hina ka-sha-n-man
enfermo-PRT-RES ser/estar-PROG-3-COND
'Parece que está enferma.'

El marcador -chus hina/chu shina significa aproximadamente 'creo', 'pienso', 'al parecer' (I guess, I think, apparently, véase Faller 2006: 3), y se puede usar si el hablante tiene evidencia directa, pero parcial, para respaldar la aseveración.

Como demuestra el ejemplo (1a), en cuzco quechua, =chá no puede usarse en tales casos. Tampoco se usa si el hablante está seguro que la aseveración es verdadera o falsa, aunque haya llegado a esta conclusión a través de un proceso de razonamiento (Faller 2007: 5). Este hecho respalda el análisis de =chá en cuzco quechua como un evidencial y un modal epistémico. Para que un acto que contiene =chá sea exitoso, el hablante necesita creer en la posibilidad de la proposición, y tiene que haber llegado a esta conclusión por su propio razonamiento. En la Sección 4.2, describiré en más detalle la distribución y el significado del enclítico =cha en tena kichwa, demostrando que su semántica difiere bastante de la descrita arriba para su cognado en cuzco quechua. 
El tercer enclítico que aparece es el reportativo =si. Sin embargo, no le describiré aquí en más detalle, ya que los datos recaudados hasta el momento indican que tena kichwa no tiene un marcador evidencial reportativo. Lo mismo ocurre en algunos otros dialectos, como el imbabura quechua (QII), hablado en la región Andina de Ecuador, donde, al igual que en tena kichwa, los relatos verbales se indican de manera perifrástica con el verbo ni('decir', Cole 1982: 164-5).

\subsection{Los enclíticos 'evidenciales' en tena kichwa}

En la sección anterior se ha presentado el paradigma evidencial atestiguado mayoría de los dialectos quechuas. En lo que sigue, explicaré que aunque en tena kichwa existen los enclíticos =mi y =cha, cognados del evidencial directo e inferencial/ conjetural, respectivamente, no se pueden analizar cómo evidenciales en este dialecto. Demostraré que en tena kichwa dichos enclíticos más bien deben analizarse como marcadores de autoridad epistémica del hablante o falta de ella (véase Sección 2).

Como se ha mencionado anteriormente, en la mayoría de los dialectos quechuas $=m i$ se ha analizado como evidencial directo, o como marcador del 'mejor fundamento posible'. Sin embargo, en tena kichwa no está limitado solamente a oraciones basadas en evidencia directa y el 'mejor fundamento posible'. Considérese:

(2) Evidencia directa

Tamyawmi

tamya-w $=\mathbf{m i}$

llover $-\mathrm{PROG}=\mathbf{m i}$

'Está lloviendo'

Contexto: El hablante ve que está lloviendo.

el_21092014_010035

El uso de =mi en (2) es compatible con su interpretación como un evidencial directo o marcador de 'mejor fundamento posible'. Sin embargo, =mi también puede ocurrir en oraciones que se basan en evidencia indirecta:

(3) Inferencia/conjetura

[Cesar] mingamami rishka.

[Cesar] minga - ma $=\mathbf{m i}$ ri -shka

[Cesar] trabajo.comunitario-DAT=mi ir -ANT

'[Cesar] ha ido a la minga'

Contexto: El hablante llega a casa de Cesar. Él no está ahí, y es el día de la 'minga' en la comunidad. El hablante sabe que Cesar siempre participa en estos eventos.

el_21092014_01051

\footnotetext{
${ }^{5}$ Cada ejemplo está acompañado por su fuente: el nombre del archivo en el corpus y el número de oración. En el caso de que un ejemplo provenga de textos sin grabar, se describe como 'obtenido'.

'Minga es el termino kichwa que se usa para trabajo comunitario. La persona que convoca la minga asegura comida y bebida para el día, y todos los vecinos viene a ayudar. Este tipo de colaboración se utiliza por ejemplo para construir casa u otros edificios, cortar grandes cantidades de madera, o realizar trabajos que benefician a toda la comunidad, por ejemplo pintar la iglesia o escuela local.
} 
(4) Inferencia/conjetura

Nuka yaya shamuwmi yachin.

ñuka yaya shamu $-\mathrm{w} \quad \mathbf{= m i}$ yachi-n

1SG padre venir $-\mathrm{PROG}=\mathbf{m i}$ parecer -3

'Mi padre ya viene, me parece.'

Contexto: El hablante oye pasos afuera. Desde hace un tiempo estaba esperando la llegada prevista de su padre.

el_21092014_01035

Las oraciones en los ejemplos (3) y (4) están basadas en evidencia inferencial u conjetural. Como se ha mencionado en la Sección 4.1, en los otros dialectos quechuas las aseveraciones basadas en inferencia o conjetura no admiten el $=m i$, sino el $=$ cha.

Cabe explicar en más detalle el uso de $=m i$ con el modal epistémico yachin (parecer-3, 'parece') en el ejemplo (4) va en contra del interpretación del marcador como el evidencial directo, o como el marcador del 'mejor fundamento posible'. Como he explicado en la Sección 4.1, el uso de =mi como marcador del 'mejor fundamento' solo es posible si el hablante cree que posee la mejor evidencia, y si la aseveración no se basa en evidencia directa parcial. En este caso, el uso del modal epistémico atenúa la aseveración, indicando que el hablante no cree las evidencias de las que dispone son conclusivas. Además, el hecho de que el padre viene es un evento observable. Por lo tanto, evidencia auditiva cuenta como evidencia directa, pero solo parcial. Si el hablante se siente en derecho de concluir que su padre viene, es porque conoce las rutinas de su padre o el ritmo de sus pasos. Por lo tanto, el uso de $=m i$ ejemplificado en (4) se basa en una inferencia, y no en acceso directo a la información. Como se ha expuesto anteriormente, esta base evidencial no sería adecuada para el uso de =mi en otros dialectos quechuas.

A diferencia de lo que ocurre muchas otras lenguas quechuas, en tena kicha =mi también puede usarse con información reportativa. Considérese:

(5) Evidencia reportativa

[Rimawanun] Saida ungushkami sirik nisha.

[rima-wa -nun] Saida ungu -shka $=\mathbf{m i}$ siri-k ni -sha say -1OBJ-3PL NOMBRE enfermarse-ANT=mi quedarse-NMLZ decir-COR '[Me dicen que] Saida está enferma'

Contexto: La hablante repite lo que ha escuchado de otros sobre Saida, sin haber visto a/hablado con la enferma.

el_25092014_01 113

El ejemplo (5), basado en un relato verbal, presenta una estrategia reportativa donde $=m i$ se usa junto al verbo ni- ('decir') para introducir una aseveración hecha por otra persona. En este caso, nisha (decir-COR) es un marcador reportativo, desprovisto de su contenido semántico original. Eso se evidencia en su uso junto a otro verbo de habla, rima- ('decir'), ya que si ambos verbos conservarían su semántica original, oración (5) produciría una redundancia ('Me dicen que Saida está enferma diciendo'). 
Como hemos visto, en cuzco quechua la información reportativa se introduce con el reportativo $-s i$, y no con la construcción perifrástica $=m i+n i-$. Sin embargo, algunos dialectos, por ejemplo imbabura quechua, también usan el verbo ni- para introducir información reportativa (Cole 1982: 164-65). Mientras en tena kichwa no se atestigua un cognado del reportativo -si, este sí ocurre en el dialecto de Imbabura, pero no marca un relato, sino una especulación (Cole 1982: 165).

Lo importante desde el punto de vista del análisis desarrollado en este artículo es que en ejemplos como la oración (5) $=m i+n i$ - introduce las palabras de otra persona, pero no indica que el hablante las evalúe como verdaderas. Considérese:

(6)

Chi churiwa.... ñuka iyakani churiwami....

chi churi -wa ñuka iya -ka -ni churi -wa $=\mathbf{m i}$

DEM.D hijo -DIM 1SG pensar-PST-1 hijo -DIM =mi

paywa churi manga nisha...

pay -pa churi=mi a -nga ni -sha

3SG-GEN hijo $=\mathbf{m i}$ COP-FUT decir - COR

'Este niño, he pensado [que era] el hijito [del campesino], este ha de ser su hijo

[dije]'

Contexto: La hablante se da cuenta que estaba equivocada.

el_21092014_02069

En (6), la hablante hace un comentario sobre un video que ha visto ('La Historia de las Peras', Pear Story, Chafe 1980, véase también la Sección 5.1). Admite que estaba equivocada pensando que uno de los protagonistas era hijo del otro, mientras tan solo era un chico que ha venido a robar algo de fruta. Sin embargo, aun sabiendo que su opinión estaba equivocada, usa la construcción $=m i+n i$ - en la segunda cláusula del ejemplo.

Los ejemplos (3), (4), (5) y (6) demuestran que el significado del =mi en tena kichwa es más amplio que el significado del evidencial directo, o bien del marcador de 'mejor fundamento posible'. La interpretación del $=m i$ como un enclítico que indica el 'mejor fundamento posible' se ve cuestionada también por el ejemplo (7):

(7) Adivinanza

Llukipuramami rin, llukipurama...

lluki -pura-ma $=\mathbf{m i}$ ri $-\mathrm{n}$ lluki $\quad$-pura-ma

izquierda-lado-DAT=mi ir -3 izquierda-lado-DAT

'[la bolita] va a la izquierda, a la izquierda'

Contexto: El hablante acaba de ver un truco de trile, pero no sabe dónde está la bolita y lo está adivinando.?

\footnotetext{
${ }^{7}$ Estos datos provienen de un experimento: dos hablantes han mirado seis grabaciones de un mago demostrando juegos de trile. La primera vez que miraban cada juego, el video estaba cortado de tal manera que no revelaba la localización final de la bolita. Entonces les pedía que adivinasen la localización de la bolita. Luego miraban el juego completo con el final, y podían comparar sus suposiciones con la verdadera localización de la bolita. El video, audio y transcripción de la sesión
} 
Recordemos que si =mi fuera el marcador del 'mejor fundamento posible', podría usarse sólo en el caso de que el hablante estuviera seguro de tener la mejor fuente de información (véase arriba y Sección 4.1). Eso no ocurre en la oración (7), ya que esta se basa en una suposición del hablante sobre el resultado de un juego de azar. En la oración (7), el hablante adivina la localización de la bolita en el trile. La oración forma parte de una serie de adivinanzas, y en ningún caso previo su suposición sobre la localización de la bola ha sido correcta. Por lo tanto, en el caso de (7) el hablante no tiene ningún motivo para suponer que lo que dice se basa en la mejor evidencia posible - la proposición que expresa es pura conjetura, y aun así está marcada con =mi. La escasa evidencia de la que dispone el hablante en este caso podría considerarse como evidencia directa, pero parcial, ya que el hablante no ha visto aún donde ha parado la bolita. Como he expuesto anteriormente, si $=m i$ en tena kichwa fuera el marcador del mejor fundamento posible, no podría usarse en tal caso (véase ejemplo (1) en la Sección 4.1).

Cabe destacar que la oración (7) difiere de una adivinanza a ojos cerrados. La evidencia visual parcial que adquiere el hablante le permite usar $=m i$, ya que le da el fundamento para creer que posee la autoridad epistémica necesaria para determinar la localización de la bolita. Si el hablante tuviera que cerrar los ojos, perdería el respaldo tanto para su aseveración, como para sostener la autoridad epistémica. Por lo tanto, supongo que en el caso de adivinanza a ojos cerrados no sería posible el uso de $=m i$, aunque está suposición ha de confirmarse con los hablantes. ${ }^{8}$

Los ejemplos citados arriba pueden explicarse de una manera uniforme si analizamos el enclítico $=m i$ en tena kichwa no como un evidencial directo/marcador de 'mejor fundamento', pero como un marcador de la autoridad epistémica del hablante. La semántica de $=m i$ no es el tema principal de este artículo, y por lo tanto solo la describiré aquí en términos muy generales. Más detalle sobre la semántica del $=m i$ y la autoridad epistémica puede encontrarse en Grzech (2016a; 2016b). El concepto de 'autoridad epistémica' es más subjetivo que el de 'evidencia directa', e incluso el de 'mejor fundamento posible'; Como se ha visto arriba, para afirmar que posee autoridad epistémica, el hablante no necesita respaldo de un tipo determinado de evidencia, ni convicción de que la evidencia que tiene es la mejor posible. Basta con que el hablante se sienta con derecho a opinar, y que perciba que la aseveración marcada con $=m i$ está dentro de su 'territorio de información' (Kamio 1997, véase Sección 2). Además, en tena kichwa $=m i$ suele usarse cuando el hablante supone que el conocimiento del oyente es inferior al suyo (Grzech 2016a; 2016b). Sin embargo, las condiciones bajo las cuales el hablante llega a esta evaluación requieren investigación más detallada, debido a su alto grado de subjetividad.

La interpretación de =mi como marcador de autoridad epistémica exclusiva del hablante se ve respaldada por la asociación de $=m i$ con el foco. Por su escasa frecuencia - menos

están disponibles en línea, en el Archivo de Lenguas en Peligro de Extinción Engangered Languages Archive (ELAR, URL de la sesión: https://elar.soas.ac.uk/Record/MPI1034554).

${ }^{8}$ Gracias al revisor anónimo por señalar la diferencia entre este ejemplo y una adivinanza sin fundamento sensorial . 
de $6 \%$ de las oraciones ${ }^{9}$ - el enclítico no se puede considerar como un marcador del foco, aunque todas sus ocurrencias están asociadas con el contenido focal. Sin embargo, el único contexto en el cual $=m i$ es requerido para que el acto sea afortunado es el del foco correctivo (véase Grzech 2016a: cap. 4). Considérese:

(8)

Mana ñuka ushichu, ñuka warmimi / \#warmi.

mana ñuka ushi=chu, ñuka warmi=mi. / \#warmi

NEG 1SG hija=Q/NEG 1SG mujer=mi / \#mujer

'[Ella] no [es] mi hija, [es] mi mujer.'

el_28112014_05

En (8), el hablante corrige al oyente, quien ha pensado que la mujer joven que acompaña al hablante es su hija. El hecho de que en este contexto la misma oración sin =mi sería desafortunada indica que la semántica del marcador está asociada no solo con la autoridad epistémica, como se ha visto en los ejemplos anteriores, pero también con el hecho que el hablante evalúa la información marcada por =mi como nueva o inesperada para el oyente.

Los ejemplos citados arriba demuestran también que en tena kichwa, la evidencia conjetural o inferencial no se indica con $=c h a$, como en otros dialectos quechuas. De hecho, cuando he presentado a mis colaboradores oraciones inferenciales o conjeturales marcadas con =cha, las han juzgado como actos desafortunado. Han explicado que oraciones que contienen =cha deberían interpretarse más bien como preguntas:

(9) =cha : uso desafortunado en una oración afirmativa

a. \#tamia-shka =cha

llover-ANT=cha

Significado intencionado: 'Ha llovido/Tenía que haber llovido'

Contexto: El hablante no ha visto la lluvia, pero ve que la tierra está mojada.

b. Tamiashkacha?

tamia-shka =cha

lluvia-ANT=cha

'¿Habrá llovido? / 'Ha llovido, ¿no?'

Aunque los hablantes rechazaron oraciones afirmativas con =cha cuando se las he presentado para evaluación, en el discurso natural =cha sí ocurre en oraciones que no son interrogativas. Se ha atestiguado su uso en preguntas retóricas - como en (10) - y oraciones dubitativas - como en (11):

(10) Pregunta retórica

Chiraygucha kay islamaga allí...

chi-raygu =cha kay isla-ma=ga alli

\footnotetext{
${ }^{9}$ En la parte anotada del corpus, que incluye 1537 turnos conversacionales, =mi ocurre 92 veces.
} 


\section{normas}

Autoridad epistémica y atenuación en Tena Kichwa | Karolina Grzech

DEM.D-CAUS=cha DEM.P orilla-DAT=TOP bueno/a

'Será por eso que [la tierra] en la orilla es tan buena [para cultivar]'

Contexto: Dos agricultores hablan de la calidad de la tierra que hay alrededor de su aldea. El hablante hace un comentario sobre el lote de tierra de la oyente.

in_01082013_02094

(11) Oración dubitativa

Ima shutiracha, Shangricha nijkuna akay...

ima shuti-ta =cha Shangri=cha ni-k-kuna a-ka=y

que nombre-ACC=cha NOMBRE=cha decir-NMZL-PL AUX-PST=EMPH.INT

'Como era su nombre, será que le llamaban Shangri...'

Contexto: Una hablante mayor intenta acordarse de cómo se llamaba un chamán al que

conocía en su juventud.

in_26052013_02 132

Los ejemplos (10) y (11) demuestran que =cha en tena kichwa no ocurre en los mismos contextos que sus cognados en otros dialectos quechuas. En la Sección 4.1 he mencionado que en cuzco quechua el evidencial inferencial/conjetural no se puede usar si el hablante tiene evidencia directa, sino parcial. Sin embargo, el ejemplo (11) demuestra que el uso de =cha es posible en este contexto en tena kichwa, y el ejemplo (7) ha demostrado que en tal caso también se puede usar el marcador $=m i$.

En el ejemplo (11), una hablante mayor intenta acordarse del nombre de una persona que conocía en su juventud. Por lo tanto, tiene para su suposición evidencia directa - su memoria - pero parcial, ya que no se acuerda bien del nombre de la persona. También el ejemplo (10) demuestra que el análisis del =cha en tena kichwa como un evidencial inferencial/conjetural es problemático. En este caso, el hablante tiene evidencia directa para la proposición que expresa, ya que él también tiene un lote de tierra en la orilla. Por lo tanto, este ejemplo no se puede explicar con el análisis evidencial. El enclítico =cha tampoco se suele usar en oraciones conjeturales o inferenciales. En estos contextos los hablantes prefieren la construcción $=m i+$ yachin $(=m i+$ parecer -3 , véase ejemplo (4)), o solo yachin (parecer-3). En el experimento con las grabaciones de los juegos del trile, descrito en la Sección 4.1, los hablantes no han usado =cha ninguna vez en 10 minutos (113 turnos) de conversación, mientras en otras variedades quechuas esta hubiera sido la estrategia habitual para indicar adivinanza (véase Faller 2002; 2007). Más bien, los hablantes del tena kichwa han usado =mi y/o yachin.

Las características de la distribución de =cha en tena kichwa, mencionadas arriba, indican que en este dialecto el enclítico no se puede analizar como un evidencial conjetural o inferencial. Sin embargo, los usos de =cha en (10) y (11) se pueden explicar si el enclítico se analiza como un marcador de falta de autoridad epistémica por parte del hablante. En la siguiente sección explicaré este análisis en más detalle.

\section{LOS USOS DISCURSIVOS DEL ENCLÍTICO =CHA}

En la sección anterior he indicado que la semántica de los enclíticos =mi y =cha en tena kichwa difiere de la de sus cognados en otras lenguas quechuas. En esta sección, me 
enfocaré en la semántica y el uso discursivo de =cha. En primer lugar, esbozaré la relación del enclítico con el concepto de 'falta de autoridad epistémica' (5.1). Por consiguiente, propondré un análisis de =cha según el cual sus usos discursivos puedan considerarse como ejemplos de atenuación (5.2).

\section{1 =cha y falta de autoridad epistémica}

Como he explicado en la sección anterior, =cha en el tena kichwa no es un marcador evidencial inferencial/conjetural. En esta sección demostraré que el enclítico puede analizarse como un marcador de falta de autoridad epistémica, o sea como un marcador que indica que el hablante considera no tener el derecho a saber o sostener algo (véase Sección 2). Al admitir que no tiene la autoridad epistémica, el hablante reconoce que una aseveración se encuentra fuera de su 'territorio de información' (Kamio 1997): el conjunto de conocimiento sobre el cual se siente en derecho a opinar. En esta sección expondré que para hacer explicita su falta de autoridad epistémica, los hablantes del tena kichwa pueden optar por usar en enclítico =cha. Cabe destacar que al igual que $=m i$, =cha es bastante poco frecuente. En la parte anotada del corpus, ocurre tan solo en $2 \%$ de los turnos conversacionales $(n=33)$.

En términos puramente teóricos, la falta de autoridad epistémica difiere de una conjetura. Al expresar una conjetura, el hablante indica que considera una determinada proposición como verosímil. En cambio, indicando la falta de autoridad epistémica, el hablante no evalúa la veracidad de la proposición: más bien admite no poder hacerlo. En otras palabras, expresando una conjetura el hablante presenta su evaluación de la realidad extralingüística. A cambio, expresando la falta de autoridad epistémica, el hablante indica que no cuenta con la información suficiente como para evaluar la veracidad de la aseveración. La expresión de falta de autoridad epistémica por parte del hablante sugiere que el oyente posee más información, o que la información pertenece a su 'territorio de información'. Por lo tanto su contribución es necesaria antes de que la aseveración puede ser aceptada como verdadera por ambos interlocutores.

La invocación al conocimiento del oyente explica, en parte (véase también Sección 5.2), porque en tena kichwa las conjeturas con =mi/yachin siempre se interpretan como oraciones declarativas, mientras que oraciones con =cha suelen - sobre todo sin el contexto - interpretarse como interrogativas:

a. Yaya yachin, paywa yaya...

yaya yachi-n, pay -pa yaya

padre parecer-3 3SG-GEN padre

'[el] padre, parece [que es] su padre...'

b. Yaya yachin, paywa yayacha...[?]

el_18092014_02 28
yaya yachi $-n$,
pay -pa
yaya $=$ cha
padre parecer-3 3SG-GEN padre=cha
'parece [que es el] padre, [será] su padre, ¿no?' 
La oración en (12a) proviene de una descripción de 'La Historia de las Peras' (Pear Story), un cortometraje diseñado para estimular oraciones en el contexto de documentación lingüística (Chafe 1980). El hablante ve a dos personajes, un hombre adulto y un niño, y describe la supuesta relación entre los dos. La oración en (12b) podría ocurrir en un contexto similar. Ambas han sido pronunciadas con entonación declarativa. Sin embargo, el ejemplo (12a) - con el modal epistémico yachin (parecer-3) - se interpreta como una proposición dubitativa. En cambio, el (12b) - con el marcador de falta de autoridad epistémica $=c h a-$ se percibe como una solicitud de confirmación de la intuición del hablante.

En base a los ejemplos citados hasta el momento podríamos preguntarnos si =cha no podría interpretarse simplemente como un marcador interrogativo o dubitativo, que solicite la confirmación por parte del oyente. Sin embargo, el enclítico ocurre también en oraciones que no se pueden considerar como interrogantes pendientes de ratificar, que podrían traducirse con la coletilla interrogativa '¿no?'. Considérese:

Mana yachani, imaracha ranga rawn...

mana yacha-ni ima $=$ ta =cha ra - nga ra $-\mathrm{w}$ - $\mathrm{n}$

NEG saber -1 que=INTER=cha hacer-FUT AUX -PROG -3

'No sé, que va a hacer...[el protagonista del historia]'

in_24092014_01 026

Maymacha rinun, payna... payna wasima rinawn yachin

may -ma =cha ri-nun payguna payguna wasi-ma ri -nun yachi $-n$ donde-DAT=cha ir-3PL 3PL.PRO 3PL.PRO casa -DAT ir -3PL parecer -3 'No sé a dónde van ellos, ellos parece que se van a casa...'

el_24092014_02 028

Los ejemplos (13) y (14) también provienen de relatos de 'La Historia de las Peras'. Ambos ponen en evidencia que el uso de =cha no se limita a marcar aseveraciones para que sean confirmadas por oyente, ni a marcar aseveraciones cuya veracidad el hablante considera dudosa.

Más bien, como he sugerido anteriormente, =cha indica que el hablante no tiene la información suficiente para evaluar la situación extra-lingüística. Este significado del =cha queda explícitamente manifestado en ejemplos (13) y (14), ya que en ambos casos el hablante admite no saber interpretar lo que había visto. Estos ejemplos también sugieren aunque se necesitan más datos para confirmar la hipótesis - que la invocación al conocimiento del oyente puede ser tan solo una extensión pragmática del =cha, ya que no ocurre en todos los casos de su uso. Este también es el caso del ejemplo (11), donde la hablante no se acuerda del nombre de un conocido, pero su interlocutor es un hombre joven que tiene aún menos acceso epistémico a la información en cuestión. Sin embargo, la semántica de 'falta de autoridad epistémica' del hablante - sin o con la invocación al oyente - es compartida por todas las ocurrencias de =cha en el corpus. 


\section{normas}

En la siguiente sección propondré una análisis de =cha en tena kichwa como una herramienta potencialmente atenuadora, explicando cómo, a través de su semántica de 'falta de autoridad epistémica', el marcador permite al hablante 'minimizar su papel en la enunciación' (Briz y Albelda 2013: 292).

\section{2 =cha como herramienta de atenuación}

En la Sección 2, he definido la atenuación como '[una] actividad argumentativa (retórica) estratégica de minimización de la fuerza ilocutiva y del papel de los participantes en la enunciación' (Briz y Albelda 2013: 292). La descripción de las propiedades y el uso de =cha provistas en las Secciones 3 y 4 demuestra que al usarlo, los hablantes se señalan faltos de autoridad epistémica. Es decir, a través del uso del enclítico el hablante se presenta como interlocutor sin 'derecho a evaluar al asunto del que habla' (Heritage y Raymond 2005: 16; Stivers et al. 2011: 11).

Por lo tanto, al usar =cha el hablante se construye a sí mismo como el interlocutor sin derecho a saber u opinar. Propongo que, a consecuencia de esta auto-presentación del hablante, =cha puede ser analizado como una herramienta que minimiza el papel del hablante en la enunciación, de modo que el efecto se su uso coincide con una de las principales funciones de atenuación señaladas por Briz y Albelda (2013). En esta sección me enfocaré en elucidar esta propuesta. Describiré el impacto de =cha sobre los diferentes aspectos de la oración, con el fin explicar cómo el uso del enclítico desencadenen el proceso de atenuación.

Antes de proceder a describir las propiedades atenuadoras de =cha en tena kichwa, cabe mencionar que los análisis de los cognados de =cha en algunas otras lenguas quechuas también han apreciado la función atenuadora del enclítico. Weber (1986) explica que =chi en huánuco quechua es un marcador de conjetura, y su uso permite evitar el compromiso con la veracidad de la oración. El autor también destaca que =chi se usa para indicar preguntas y para lograr varios efectos discursivos, tales como ironía y sarcasmo.

En su descripción de wanka quechua, Floyd sostiene que el significado prototípico del marcador $=\operatorname{chr}(a)$ es 'atenuación en el campo validacional' (Floyd 1997: 136). La función atenuante del marcador se aproxima a la de los modales epistémicos en lenguas europeas, ya que a través de su uso el hablante indica que 'no asume responsabilidad por su evaluación del a factibilidad de un evento no experimentado o no corroborado' (Floyd 1997: 136). El autor también menciona que los significados no-prototípicos del marcador tienen que ver con la 'atenuación en otros dominios', tales como 'la distancia psicológica entre el oyente y la proposición', por ejemplo en el caso de inferencias en primera persona (Floyd 1997: 139). De los efectos atenuantes descritos por Floyd, este último parece ser el que más se aproxima la indicación de falta de autoridad epistémica introducida por =cha en tena kichwa.

Como Weber (1986), Floyd (1997) indica que el marcador se usa para lograr efectos discursivos, por ejemplo ironía, y que se usa en preguntas retoricas. Como he mencionado anteriormente, el =cha en tena kichwa se puede usar en preguntas retoricas, pero no se han atestiguado los usos irónicos o sarcásticos en los datos obtenidos hasta el momento. 


\section{normas}

La 'explotación discursiva' de evidenciales indirectos para lograr una reducción de responsabilidad y/o atenuación, es un fenómeno descrito en estudios comparativos de sistemas evidenciales (véase Aikhenvald 2004; Boye 2012). Este artículo propone que la atenuación también puede estar relacionada con el uso de marcadores epistémicos noevidenciales, tales como =cha en tena kichwa. En el caso de los marcadores modales epistémicos, la atenuación es el resultado de la reticencia del hablante para aceptar la proposición como verdadera. Indicando que una proposición es verosímil, pero no necesariamente cierta, el hablante señala que la proposición no debería ser aceptada como verdadera por los participantes del intercambio comunicativo, al menos no sin más elaboración. El marcador =cha provoca un efecto parecido al señalar la falta de primacía epistémica del hablante.

Para explicar el efecto atenuante de =cha, haré uso del marco teórico que asume que la comunicación consiste en un continuo desarrollo del 'conocimiento en común' (véase Krifka 2007; Féry y Krifka 2008). El 'conocimiento en común' (Common Ground) incluye toda la información compartida entre, y considerada como verdad por los participantes del intercambio comunicativo (véase Stalnaker 1974). Este conjunto de proposiciones crece durante la comunicación, a medida que se responden las 'preguntas bajo discusión' (Questions Under Discussion, véase Asher 1993). Según esta teoría de la comunicación, cada turno conversacional sirve para responder una 'pregunta bajo discusión', o para introducir una nueva pregunta. Por ejemplo, si preguntamos a alguien: '¿Cómo se llama tu primo?', el nombre del primo de nuestro oyente se convierte en la 'pregunta bajo discusión'. Cuando el oyente responde: 'Se llama Alberto', responde a la pregunta, y el nombre del primo pasa a formar parte de los 'conocimientos en común' compartido por los hablantes.

Si percibimos la comunicación como una expansión del 'conocimiento en común' a través de la provisión de respuestas a las 'preguntas bajo discusión', la función del =cha consiste en indicar que dada oración no debería considerarse como una respuesta satisfactoria a la actual 'pregunta bajo discusión'. La falta de autoridad epistémica codificada por =cha señala que una contribución adicional es necesaria para responder a la pregunta, ya que el hablante no dispone de un acceso epistémico suficiente para responderla de manera satisfactoria. Eso explica porque, como se ha demostrado en el ejemplo (9), en falta de contexto adicional las oraciones con $=$ cha pueden interpretarse como interrogantes por ratificar.

Que una proposición no debe ser incluida en el 'conocimiento en común' es una instrucción para el oyente sobre cómo debe interpretar la oración. Esta instrucción impide a que el contenido informativo de la oración sea aceptado como verdadero y concluyente (desde el punto de vista de 'preguntas bajo discusión') por parte de ambos participantes del intercambio comunicativo. Por lo tanto, =cha se puede interpretar como una herramienta que rebaja la 'inmediatez comunicativa' (Briz y Albelda 2013: 292) del acto en el que ocurre. Esta función de $=$ cha es compatible con su análisis como un marcador atenuante.

Sin embargo, este no es el único efecto producido por el uso de =cha que puede considerarse atenuador. La presencia del marcador en una oración también produce otro resultado: distancia al hablante de la oración. Este distanciamiento se debe a lo señalado anteriormente: el hablante indica no tener acceso epistémico suficiente para poder 
pronunciarse con autoridad sobre la proposición expresada por la oración. La información marcada con el enclítico no pertenece al 'territorio de información' del hablante. El enclítico =cha codifica esta distancia epistémica, que, aunque pueda existir también en caso de oraciones no marcadas con =cha, cuando se usa el marcador queda declarada explícitamente. A diferencia de los modales epistémicos, en este caso el distanciamiento del hablante de la aseveración no tiene que ver con la reducción de su compromiso con la veracidad. Más bien, como se ha mencionado anteriormente, el distanciamiento se debe al hecho de que el hablante reconoce su falta de capacidad de evaluar a la veracidad de la oración. El efecto comunicativo logrado por este distanciamiento puede considerarse atenuativo, ya que reduce la fuerza comunicativa de la oración marcada; a través del uso de =cha, el hablante señala de que su contribución comunicativa no es de suficiente calidad como para resolver la actual 'pregunta bajo discusión'.

Como se ha explicado arriba, esta insuficiencia informativa de las oraciones marcadas por =cha muchas veces da lugar a su interpretación interrogativa por parte del oyente. Sin embargo, no es evidente a qué aspecto de la fuerza comunicativa influencia el enclítico =cha. Caffi (1999: 884) señala que la atenuación puede basarse en tres niveles de la oración: la proposición, la ilocución, y la fuente de la oración. Los datos del tena kichwa sugieren que =cha no contribuye al contenido proposicional de la oración, ya que su contribución a la oración no puede ser negada. No obstante, este análisis debería corroborarse con más datos (véase Grzech 2016a: cap. 5). En la Sección 4.2 he demostrado que $=$ cha no es un evidencial. Por lo tanto, tampoco opera al nivel de la fuente de la oración. Sin embargo, el corpus disponible y los análisis llevados a cabo hasta el momento no permiten establecer si =cha opera al nivel de la ilocución, o al nivel de 'fuerza sentencial' (sentential force, véase Portner 2006). Esta última está relacionada con la forma gramatical de la proposición, y no - como la fuerza ilocutiva - con el intencionado efecto comunicativo de la oración. Ya que Caffi (1999) no considera la dimensión de 'fuerza sentencial' en su trabajo, se necesita más investigación para determinar el nivel de la oración al que influencia el =cha para lograr el efecto atenuador.

A cambio, los datos disponibles permiten determinar con certeza que el =cha sólo consigue la atenuación de hablante (véase Briz y Albelda 2013). Los marcadores epistémicos en muchas lenguas del mundo se someten al 'cambio de origo' (origo shift): en las oraciones declarativas se asocian con el hablante, y en las oraciones interrogativas - con el oyente (véase por ejemplo Boye 2012). Este proceso se aplica por ejemplo al enclítico =mi en TK, como lo demuestran los ejemplos (15) y (16):

Ñuka shuti anmi Karolina.

ñuka shuti an=mi Karolina

1SG nombre ser/estar=mi NOMBRE

'Mi nombre es Karolina.'

obtenido

Ima shutimi?

ima shuti=mi 


\section{normas}

\section{que nombre $=\mathbf{m i}$}

'¿Cómo se llama?'

Contexto: La hablante pregunta al oyente por el nombre de una tercera persona.

En el ejemplo (15) la hablante se presenta al oyente diciendo su nombre. La fuente de la primacía epistémica en este caso es claramente la hablante. En (16), la hablante pregunta al oyente por el nombre de una tercera persona, a la que sólo conoce el oyente. Por lo tanto, en esta oración =mi señala la primacía epistémica del oyente, y no de la hablante una situación opuesta a la que ocurre en (15). El cambio de origo no ocurre en el caso del =cha, como lo demuestran los datos presentados en la Sección 4.2. Tanto como cuando ocurre en oraciones que se interpretan como declarativas, como en oraciones interrogativos, =cha señala al hablante. Considérese:

$$
\begin{aligned}
& \text { Ukllarishka washa payna makira waktanawn, } \\
& \text { ukllari-shka washa payguna maki-ta wakta-nun } \\
& \text { abrazar-ANT después 3PL mano -ACC chocar-3PL } \\
& \text { imarashacha waktanun mana... mana yachani } \\
& \text { imarasha=cha wakta-nun mana mana yacha-ni } \\
& \text { por.qué =cha chocar }-3 P L \text { NEG NEG saber }-1
\end{aligned}
$$

'Después de abrazarse se chocan las manos [chocan los cinco], por qué chocarán así... no lo sé'

$(18)=(9 b)$

Tamiashkacha?

tamia-shka =cha

Iluvia-ANT=cha

¿¿Habrá llovido?' / 'Ha llovido, ¿no?’

En el ejemplo (17) =cha marca una pregunta retórica dentro de una oración declarativa, donde el hablante admite no saber porque las personas que ha visto en el video se chocan las manos. La oración en (18) en falta de más contexto sea siempre interpretada como una oración interrogativa, donde la falta de autoridad epistémica indicada por el enclítico también corresponde al hablante. ${ }^{10}$ Esta situación es opuesta a la que hemos visto en el caso de los ejemplos (15) y (16). Entonces podemos concluir que el cambio del origo ocurre en el caso del $=m i$, pero no en el caso del $=c h a$.

\footnotetext{
${ }^{10}$ Cabe destacar que las traducciones al español en los dos últimos ejemplos, y de hecho en todos los ejemplos en este artículo, no debería tomarse como una traducción literal de los ejemplos en kichwa. Que algunas oraciones admitan una interpretación variable en español solo demuestra que el significado de =cha (y de =mi) no tiene un equivalente adecuado entre las expresiones epistémicas disponibles para los hispanohablantes.
} 


\section{normas}

Los datos analizados hasta el momento no permiten establecer por qué =cha no incita el cambio del origo. Sin embargo, la falta de dicho cambio no es un fenómeno sin precedentes. En algunos sistemas evidenciales de las lenguas tibetanas, los marcadores también permanecen relacionados con el hablante independientemente del tipo del acto ilocutivo (Manuel Widmer, comunicación personal, 17 de Marzo 2016).

En esta sección he explicado el funcionamiento de =cha en tena kichwa como una herramienta de atenuación que distancia al hablante de la proposición expresada y reduce la inmediatez comunicativa de la oración. He indicado que =cha contribuye a reducir la fuerza comunicativa de la oración. El enclítico tiene este efecto, porque indica que el hablante no tiene la autoridad epistémica necesaria para proponer que una determinada proposición sea aceptada por el oyente como un elemento del 'conocimiento en común'.

Esta primera aproximación al análisis de =cha como una herramienta de atenuación no ha contado con datos suficientes para establecer de forma conclusiva en qué nivel de oración ocurre el efecto atenuador de =cha. Por lo tanto, si bien este artículo ha demostrado que =cha minimiza el rol del hablante en la oración, no ha logrado determinar si el marcador influye a la fuerza ilocutiva de la oración a la que modifica.

Lo expuesto arriba no agota las preguntas que pueden hacerse en futura investigación sobre las estrategias de atenuación en tena kichwa. El uso de =cha es sólo una de las estrategias disponibles para los hablantes de tena kichwa. Las otras, como por ejemplo el uso del verbo modal yachin (parecer-3), aún no han sido investigadas, y también queda por explorar su relación con el enclítico epistémico descrito en el presente trabajo.

Otra cuestión que queda por explorar es la relación de enclítico =cha con cortesía. Como mencionan Briz y Albelda (2013), la atenuación frecuentemente ocurre junto a las estrategias de cortesía discursiva, pero no necesariamente lo atenuado también es lo cortés. De momento no se han obtenido o analizado datos suficientes para comprobar si los hablantes del TK usan =cha para demostrar respeto a sus oyentes, o si sólo lo hacen por consideraciones relacionadas con el éxito de los actos comunicativos. Los datos necesarios para realizar este tipo de comparaciones aún están pendientes de obtener. ${ }^{11}$

\section{CONCLUSIONES}

En este artículo he descrito la semántica y los usos discursivos del enclítico =cha en Tena Kichwa. He introducido los dos enclítico 'evidenciales' atestiguados en tena kichwa - =mi $y=c h a$. He explicado que en el dialecto en cuestión deben interpretarse como marcadores de discurso relacionados con autoridad epistémica, y no como marcadores evidenciales. Por consiguiente, he desarrollado una análisis de =cha como un marcador de falta de autoridad epistémica del hablante, y he explicado como la semántica de falta de autoridad epistémica puede resultar en efectos discursivos de atenuación. He mencionado que se necesita más investigación para determinar en qué nivel discursivo opera =cha, pero los

\footnotetext{
${ }^{11}$ Un posible experimento podría incluir dos grupos: (1) personas con posiciones sociales asimétricas (estudiantes con profesores, padres con niños, empleados con patrones); (2) personas con posiciones sociales simétricas (compañeros de trabajo o colegio, maridos y mujeres). En ambos grupos, podrían crease situaciones donde uno de los participantes tendría más primacía epistémica con respecto a una determinada información que el otro. Luego las conversaciones entre los miembros de los dos grupos podrían compararse para buscar patrones en los usos de =cha.
} 


\section{normas}

datos demuestran que su ocurrencia no afecta al contenido proposicional de la oración. También he indicado que =cha sólo tiene la capacidad de atenuar a nivel del hablante, ya que sin importar el tipo de acto ilocutivo, siempre reduce la primacía epistémica del hablante, y nunca la del oyente.

Como se ha demostrado en este artículo, el efecto atenuador de =cha es más sutil que los efectos atenuadores que se logran a través del uso de marcadores evidenciales o modales epistémicos en otras lenguas, incluyendo otros dialectos quechuas. El enclítico distancia el hablante de la oración, pero su efecto no está relacionado con la reducción el compromiso del hablante con la veracidad de la aseveración. Más bien, =cha demuestra que el hablante no tiene la información necesaria como para poder evaluar la veracidad de la información. Por lo tanto, el marcador indica que se requiere información adicional antes de que los interlocutores puedan aceptar la información en cuestión como verdadera.

Cabe destacar que la sutileza de su efecto no invalida el análisis de =cha como una herramienta de atenuación. Más bien, considerar a =cha como marcador de atenuación abre nuevas preguntas dentro del estudio de la misma. El ejemplo de =cha demuestra que existen varias maneras de reducir el papel del hablante en la enunciación y distanciarlo de la proposición. Reducir el compromiso con la veracidad es una de ellas, pero los datos provenientes de tena kichwa parecen demonstrar que indicar la falta de autoridad epistémica logra el mismo fin, aunque con efectos semánticos y discursivos ligeramente diferentes.

A consecuencia, podríamos preguntarnos si y como nuestra manera de conceptualizar los procesos atenuativos podría verse influenciada por el estudio de lenguas con paradigmas epistémicos poco habituales desde el punto de vista europeo (véase por ejemplo Kunchel 2017, Schultze-Bernt 2017). Los datos de tena kichwa analizados de este artículo parecen indican que inclusión de nuevos idiomas podría implicar ciertos ajustes conceptuales, al menos en cuanto a lo que significa minimizar el papel del hablante en el discurso.

\section{REFERENCIAS}

Adelaar, Willem F. H. (1977): Tarma Quechua: grammar, texts, dictionary, Lisse, The Peter de Ridder Press.

Adelaar, Willem F. H. (1997): Los marcadores de validación y evidencialidad en quechua: ¿automatismo o elemento expresivo?, Amerindia 22, 3-13.

Adelaar, Willem F. H. y Pieter C. Muysken (2004): The Languages of the Andes. Cambridge, Cambridge University Press.

Aikhenvald, Alexandra Y. (2004): Evidentiality, Oxford, Oxford University Press.

ANCE, Asamblea Nacional Constituyente de Ecuador (2008): Constitución de la República del Ecuador. [en línea] http://02a045b.netsolhost.com/legislacion/normativa/leyes/constitucion2008.pdf [consulta: 26/01/2013].

Asher, Nicholas (1993): Reference to Abstract Objects in Discourse, Berlin, Springer Science \& Business Media.

Bergqvist, Henrik. 2017. The role of "perspective" in epistemic marking. Lingua 186-187. 5-20. doi:10.1016/j.lingua.2015.02.008.

Boye, Kasper (2012): Epistemic Meaning, A Crosslinguistic and Functional-Cognitive Study, Berlin, Boston, De Gruyter Mouton.

Briz, Antonio y Marta Albelda (2013): Una propuesta teórica y metodológica para el análisis de la atenuación lingüística en español y portugués. La base de un proyecto común (ES.POR.ATENUACIÓN), Onomázein Revista de lingüística, filología y traducción 28, 288-319, doi:10.7764/onomazein.28.21.

Caffi, Claudia (1999): On mitigation, Journal of Pragmatics 31(7), 881-909. doi:10.1016/S03782166(98)00098-8. 
Chafe, Wallace L. (1980): The Pear Stories: Cognitive, Cultural and Linguistic Aspects of Narrative Production, Norwood, N.J: Ablex.

Cole, Peter (1982): Imbabura Quechua: A Descriptive Grammar, en Bernard Comrie, Norval Smith y Anna de Haas, eds., Vol. 5, (Lingua Descriptive Studies). Amsterdam: North-Holland.

Cusihuamán, Antonio G. (1976/2001): Gramática quechua: Cuzco-Collao, 2nda edición, Cusco: Centro de Estudios Regionales Andinos "Bartolomé de Las Casas".

Evans, Nicholas R., Henrik Bergqvist y Lila San Roque. En Prensa, The grammar of engagement. Language and Cognition, UK Cognitive Linguistics Association, Cambridge: Cambridge University Press.

Faller, Martina T. (2002): Semantics and Pragmatics of Evidentials in Cuzco Quechua. Tesis doctoral sin publicar, Stanford: Stanford University.

Faller, Martina T. (2006): Evidentiality and Epistemic Modality at the Semantics/ Pragmatics Interface, University of Michigan [en línea] http://web.eecs.umich.edu/ rthomaso/lpw06/fallerpaper.pdf [consulta:21/04/2016].

Faller, Martina T. (2007): The Cuzco Quechua Conjectural. Epistemic modal or evidential? Or both?, Universidad de Sao Paulo.

Féry, Caroline y Manfred Krifka (2008): Information structure: Notional distinctions, ways of expression. En Piet van Sterkenburg, ed., Unity and Diversity of Languages, 123-135, Amsterdam, John Benjamins.

Floyd, Rick (1997): La estructura categorial de los evidenciales en el quechua wanka. (Serie Lingüística Peruana), Lima, SIL International.

Grzech, Karolina (2016a): Discourse enclitics in Tena Kichwa: A corpus-based account of information structure and epistemic meaning, Tesis doctoral sin publicar, Londres, SOAS, Universidad de Londres.

Grzech, Karolina (2016b): The non-evidential meaning of the Tena Kichwa "direct evidential" York Papers in Linguistics 3 (Special Issue: PARLAY Proceedings), 73-94.

Heritage, John y Geoffrey Raymond (2005): The Terms of Agreement: Indexing Epistemic Authority and Subordination in Talk-in-Interaction, Social Psychology Quarterly 68(1), 15-38.

Hintz, Daniel J. (2012): Building common ground: The evidential category of mutual knowledge. Nature of Evidentiality Conference, Universidad de Leiden.

Hintz, Daniel J. y Diane M. Hintz (2017): The evidential category of mutual knowledge in Quechua, Lingua 186187, (Essays on Evidentiality), 88-109. doi:10.1016/j.lingua.2014.07.014.

Howard, Rosaleen (2012): Shifting voices, shifting worlds: Evidentiality, epistemic modality and speaker perspective in Quechua oral narrative, Pragmatics and Society 3(2), 243-269, doi:10.1075/ps.3.2.06how.

Izquierdo Alegría, Dámaso y Bert Cornillie (2017): Evidentiality as encoding the mode of access. 50th Annual Meeting of Societas Lingustica Europea, 10-13 de Septiembre 2017, Universidad de Zurich.

Kamio, Akio (1997): Territory of Information, Amsterdam, John Benjamins.

Knuchel, Dominique (2017): Engagement in Kogi demonstratives, 50th Annual Meeting of Societas Linguistica Europea, 10-13 de Septiembre 2017, Universidad de Zurich.

Krifka, Manfred (2007): Basic notions of information structure. En Caroline Féry, Gisbert Fanselow y Manfred Krifka, eds., Interdisciplinary Studies on Information Structure (ISIS), vol. 6, 13-56, Potsdam, Universitätsverlag Potsdam.

Lefebvre, Claire y Pieter Muysken (1988): Mixed categories: nominalizations in Quechua, Berlin, Kluwer Academic Publishers.

Lewis M., Paul, ed., (2016): Ethnologue: Languages of the World. 19a edición, Dallas, Texas, SIL International.

Maschler, Yael y Deborah Schiffrin (2015): Discourse markers: Language, meaning, and context. En Deborah Tannen, Heidi Ehernberger Hamilton y Deborah Schiffrin, eds., The handbook of discourse analysis, 189221. 2nda edición, Malden, MA, John Wiley \& Sons, Inc.

Moseley, Christopher (2010): Atlas of the World's Languages in danger. 3ra edición, Paris: UNESCO Publishing.

Mushin, Ilana (2017) Whither epistemicity?, Knowling Symposium, Universidad de Helsinki, 23-25 de Agosto 2017.

Muysken, Pieter (1995): Focus in Quechua. En Katalin É. Kiss, ed., Discourse Configurational Languages, 375393, New York, Oxford University Press.

Nuckolls, Janis (2012): From quotative other to quotative self: Evidential usage in Pastaza Quichua, Pragmatics and Society 3(2), 226-242, doi:10.1075/ps.3.2.05nuc.

Nuckolls, Janis (1993): The Semantics of Certainty in Quechua and Its Implications for a Cultural Epistemology, Language in Society 22(2), 235-55.

Palmer, Frank R. (2001): Mood and Modality. 2nda edición, Cambridge, Cambridge University Press.

Parker, Gary John (1969): Ayacucho Quechua grammar and dictionary, The Hague, Paris, Mouton.

Portner, Paul (2006): Comments on Faller's Paper, Universidad de Michigan.

Sánchez, Liliana (2010): The Morphology and Syntax of Topic and Focus: Minimalist Inquiries in the Quechua Periphery, Amsterdam, John Benjamins. 
Schiffrin, Deborah (1987): Discourse Markers, Cambirdge, Cambridge University Press.

Schultze-Bernt, Eva (2017): From shared reference to shared attention: the case of Jaminjung/Ngaliwurru 'you and me', 50th Annual Meeting of Societas Linguistica Europea, 10-13 de Septiembre 2017, Universidad de Zurich.

Spencer, Andrew y Ana R. Luis (2012): Clitics: An Introduction, Cambridge, Cambridge University Press.

Stalnaker, Robert (1974): Pragmatic Presuppositions. En Milton K. Munitz y Peter K. Unger, eds., Semantics and Philosophy, 197-213, New York, New York University Press.

Stivers, Tanya, Lorenza Mondada y Jakob Steensig (2011): Knowledge, morality and affiliation in social interaction. En Tanya Stivers, Lorenza Mondada y Jakob Steensig, eds., The Morality of Knowledge in Conversation, 3-24, Cambridge: Cambridge University Press.

Torero, Alfredo (1964): Los dialectos quechuas. Anales Científicos de la Universidad Nacional Agraria, Lima, 446-78.

Traugott, Elizabeth Closs (2010): (Inter)subjectivity and (inter)subjectification: A reassessment. En Kristin Davidse, Lieven Vandelanotte y Hubert Cuyckens, eds., Subjectification, Intersubjectification and Grammaticalization, vol. 66, 29-74. Berlin, New York, De Gruyter Mouton.

Weber, David (1986): Information perspective, profile, and patterns in Quechua. En Wallace Chafe y Johanna Nichols, eds., Evidentiality: the linguistic coding of epistemology, 137-55, Norwood, NJ, Ablex.

Willett, Thomas (1988): A Cross-Linguistic Survey of the Grammaticization of Evidentiality, Studies in Language 12(1), 51-97. 